\title{
HORST STEIBLE
}

Legitimation von Herrschaft im Mesopotamien des 3. Jahrtausends v. Chr. 


\section{Legitimation von Herrschaft im Mesopotamien des 3. Jahrtausends v. Chr.}

Es ist das Anliegen dieser Untersuchung, aus der Sicht eines Sumerologen, dessen Arbeitsgebiet die Texte des 3. Jahrtausends v.Chr. bilden, einen fachspezifischen Beitrag zur Problematik der Legitimation von Herrschaft im Alten Mesopotamien zu liefern.

Bei den Quellen, die das für diese Fragestellung relevante Material darstellen, werden die originalen, von Herrschern abgefaßten Bau- und Weihinschriften zugrunde gelegt, die Verwaltungs- und Rechtsurkunden dagegen nicht berücksichtigt. Diese sogenannten >Königsinschriften< werden hier befragt von der altsumerischen Zeit ${ }^{1}$ über die Akkad-Zeit ${ }^{2}$ bis in die neusumerische Periode, ${ }^{3}$ wobei die Schwerpunkte in der altsumerischen und altakkadischen Zeit liegen. Aus Gründen der Fundzufälle stehen dabei die Texte aus Lagaš-Girsu im Vordergrund.

Diese Texte enthalten drei verschiedene Titel für die Bezeichnung eines Herrschers: en, lugal und ensi ${ }_{2} \cdot{ }^{4}$ en ist ein Titel, der in Uruk beheimatet und dort zum ersten $\mathrm{Mal}$ in historischen Inschriften um die Mitte des 3. Jahrtausends nachzuweisen ist, dessen Keilschrift- und älteres Bildzeichen sich jedoch bis in die Uruk-Zeit des späten 4. Jahrtausends v. Chr. zurückverfolgen läßt. ${ }^{5}$ Die akkadischen Entsprechungen dazu sind allgemein bēlum »Herr« und in Südmesopotamien auch enum »En-Priester«. Hier wird dieser Titel

1 H. Steible (1982), Die altsumerischen Bau- und Weihinschriften, in: FAOS 5; J.S. Cooper (1986), Sumerian and Akkadian Royal Inscriptions $I$.

2 I.J. Gelb/B. Kienast (1990), Die altakkadischen Königsinschriften des dritten Jahrtausends v. Chr., in: FAOS 7; D. Frayne (1993), Sargonic and Gutian Periods (2334-2113 BC), in: RIME 2.

3 I. Kärki (1986), Die Inschriften der Dritten Dynastie von Ur, in: StOr 58; H. Steible (1991), Die neusumerischen Bau- und Weihinschriften, in: FAOS 9.

4 Zu diesen Titeln vgl. zuletzt Th. Jacobsen (1991), «The term ensi $*$, in: Aula Orientalis, 9, S. 113-121; dazu jetzt G.I. Selz (1998), „Über Mesopotamische Herrschaftskonzepte», S. 284-286.

5 Siehe S. 84 mit Anm. 77. 
durchgängig mit »Herr« übersetzt. Der Titel lugal, als lu ${ }_{2}$-gal »großer Mann « ${ }^{6}$ zu etymologisieren, dem im Akkadischen šarrum entspricht, wird einheitlich mit »König« übersetzt. Der Titel ensi ${ }_{2}$, mit der akkadischen Entsprechung iššiakkum, dessen Träger in der altsumerischen Zeit zum Teil auch mit dem Titel lugal nachzuweisen sind, ${ }^{7}$ wird durchweg mit $\gg S t a d t f u ̈ r s t ~{ }^{8}$ übersetzt; zumindest seit dem Ende der altsumerischen Zeit ist er aber nur noch für Statthalter in Gebrauch. ${ }^{9}$ In Verbindung mit Gottesnamen begegnet dieser Titel attributiv erweitert als ensi ${ }_{2}$-gal-G(ötter)N(ame) »Ensigal(-Priester)(von) GN $\ll_{.}^{10}$ Dies zeigt, daß der Stadtfürst zwei Aufgabenbereiche hat, einen säkularen und einen religiösen: Als alleiniger Vertreter des Stadtgottes ist er »Stadtfürst « und »oberster Priesterfürst «. ${ }^{11}$

Alle drei Titel werden also konventionell wiedergegeben, und es ist nicht das Ziel dieser Untersuchungen, die alte und lang anhaltende Diskussion darüber aufzugreifen. ${ }^{12}$

Auch auf den Aufbau des sumerisch-akkadischen Pantheons ist kurz zu verweisen. ${ }^{13}$ Für seine Ausformung sind drei Götterkreise grundlegend, die in drei zentralen Städten beheimatet waren: in Uruk mit dem Gott An als Oberhaupt, in Eridu mit dem Gott Enki an der Spitze, und in Nippur mit dem Gott Enlil an erster Stelle. Ein erstes Bemühen um eine theologische Ordnung ist in den Götterlisten von Fāra/Šuruppak (um ca. 2700 v. Chr.) ${ }^{14}$ und Tell Abū Şalābīh ${ }^{15}$ zu fassen, zu denen die gleichzeitigen Götter- bzw. TempelHymnen $\mathrm{zu}$ stellen sind. ${ }^{16}$ Doch ist durch die gesamte altsumerische Zeit hindurch in den verschiedenen Stadtstaaten das Bestreben zu beobachten, das jeweilige lokale Pantheon mit den übergeordneten Gottheiten dieser drei Götterkreise zu verbinden. ${ }^{17}$

$\mathrm{Zu}$ Beginn dieser Ausführungen seien die Gesichtspunkte genannt, die für die Legitimation von Herrschern zur damaligen Zeit ausschlaggebend waren. Zwei Arten von Legitimation sind zu unterscheiden:

„Große Person* bei W. Heimpel (1992), „Herrentum und Königtum«, in: ZA 82, S. 15f., wo ihre Rolle in der Verwaltungsstruktur von Ebla beleuchtet wird; ferner noch P. Steinkeller (1993), „Early political development in Mesopotamiak, in: Mario Liverani, Akkad, S. 124 (»the heads of tribal groupings*). Siehe etwa Akurgal und E'annatum, zwei Herrscher von Lagax, bei W. Heimpel (1992), S. 7.

8 Beachte den Vorschlag einer Grundbedeutung wefficient manager of donkeys« bei Th. Jacobsen (1991), S. 120.

9 Zuletzt W. Heimpel (1992), S. 8.

10 H. Steible (1982), S. 117.

11 Vgl. dazu zuletzt P. Steinkeller (1993), S. 117.

12 Etwa bei Th. Jacobsen (1957), »Early political development in Mesopotamia«, in: ZA 52, S. 91-140 und bei W.W. Hallo (1957), Early Mesopotamian Royal Titles, in: AOS 43.

13 Siehe Tafel II.

14 Zuletzt bearbeitet von M. Krebernik (1986), «Die Götterlisten aus Făra«, in: ZA 76, S. 161-204.

15 P. Mander (1986), Il Pantheon di Abu-Salablkh.

16 Gemeint sind die sogenannten zay-me-Hymnen, siehe dazu die Erstedition von R.D. Biggs (1974), Inscriptions from Tell Aba Salabikh, in: OIP 99,S. 45-56.

17 Für den EinfluB auf das Pantheon des Stadtstaates Lagas-Girsu siehe die zusammenfassende Graphik bei G.J. Selz (1995), Untersuchungen zur Götterwelt des altsumerischen Stadtstaates von Lagaš, S. 295. 
a) Legitimation durch Götter,

b) Legitimation durch Familienbande (Dynastiefolge) und/oder durch eigene Leistung.

Beide Arten werden zur Begründung der Vergöttlichung des Königs herangezogen. ${ }^{18}$

Aspekte, in denen sich das Bestreben um Legitimation manifestiert, sind:

Zeugung (- Geburt), Adoption, Namensnennung, Aufziehen und Ausstattung des Herrschers, Erwählung, Proklamation und Autorisation.

Für den Einstieg in die Problematik und die Demonstration der Vielseitigkeit des Themas seien hier an den Anfang gestellt die relevanten TextPassagen aus der sog. Geierstele des E'annatum, des 3. Herrschers der I. Dynastie von Lagaš, ${ }_{19}^{19}$ die aus einer bestimmten historischen Situation heraus geschaffen worden ist. Es geht dabei um den langjährigen Konflikt zwischen den Stadtstaaten Lagaš-Girsu auf der einen und dem benachbarten Umma auf der anderen Seite. ${ }^{20}$ Streitobjekt sind die Ländereien des Gu'edenna, der Kornkammer am $i_{7}$-nun(-Kanal), der gleichzeitig die Grenze zwischen beiden Stadtstaaten bildete: Es geht um die Bewässerung eines großen fruchtbaren Landstriches. ${ }^{21}$ Der aktuelle Stand war folgender: Umma hatte sich (wieder einmal) dieses Landstriches bemächtigt, aus der Sicht von Lagaš-Girsu zu Unrecht, deshalb fühlte sich der Herrscher von Lagaš-Girsu verpflichtet, die alten Grenzen wiederherzustellen. Auf die Beschwerde darüber bei dem Stadtgott von Girsu, Ningirsu, reagiert dieser mit einer Verlautbarung unbekannten Inhalts. ${ }^{22}$ Dann heißt es in

E'annatum 1 iv 9-v 17:

iv 9) [dni]n-[ $\left.\mathrm{gir}_{2}\right]-\mathrm{su}^{-\mathrm{ke}_{4}}$

10) [a]-e - - an]-na-tum-[ma]

11) $\left[\mathrm{ša}_{3}-\mathrm{g}\right] \mathrm{a}$

12) [š b]a-ni-du

13) $[\ldots .$.

14) $[\ldots .$.

15) $[\ldots .$.

16) $[\ldots .]-$.

17) mu-da-bul 2
Ningirsu

hat den E'annatum

ge-

zeugt.

[....]

[....]

[....]

[....]...

hat sich über (E'annatum) gefreut.

18 Siehe ebd., S. 17.

19 Für die Abfolge der Herrscher und ihren zeitlichen Kontext sei generell auf Tafel I verwiesen.

20 Dazu jetzt grundlegend J.S. Cooper (1983), The Lagash-Umma Border Conflict.

21 Vgl. dazu auch H.-J. Nissen (1983), Grundzüge einer Geschichte der Frühzeit des Vorderen Orients, S. 147 und jetzt zuletzt G.J. Selz (1998), S. 312 mit Anm. 149.

22 Der Text ist an dieser Stelle (Kol. iv 6-7) bis auf ein Zeichen ganz zerstört. 


\begin{tabular}{|c|c|c|}
\hline 18) & dinanna-ke $e_{4}$ & Inanna \\
\hline 19) & da mu-ni-dab & hat ihn auf den Arm genommen \\
\hline 20) & $e_{2}$-an-na- & (und) hat »Ins E'anna \\
\hline 21) & dinanna- & der Inanna \\
\hline 22) & ib-gal-ka-ka a-tum ${ }_{2}$ & von Ibgal habe ich(?) (ihn) gebracht!« \\
\hline 23) & mu mu-ni-sa & (als) seinen Namen genannt. \\
\hline 24) & dnin-bur-sag-ra & Sie hat (ihn) der Ninhursag \\
\hline 25) & $\mathrm{du}_{10}-\mathrm{zi}-\mathrm{da}-\mathrm{na}$ & auf ihren recht(mäßig)en Schoß \\
\hline 26) & mu-ni-tuš & gesetzt, \\
\hline 27) & dnin-hur-sag-ke & (und) Ninhursag \\
\hline 28) & ubur-zi-da-ne 2 & hat (ihn) an ihre recht(mäßig)e Brust \\
\hline 29) & $\mathrm{m}\left[\mathrm{u}-\mathrm{ni}-\mathrm{la} \mathrm{a}_{2}\right]$ & gelegt. \\
\hline v 1) & $e_{2}$-an-na-tum 2 & Über E'annatum, \\
\hline 2) & a-ša $a_{3}-g a-s ̌-d u_{11}-g a-$ & gezeugt \\
\hline 3) & dnin-gir $r_{2}-s u-k a-d a$ & von Ningirsu, \\
\hline 4) & dnin-gir - -su & hat sich Ningirsu \\
\hline 5) & mu-da-bul 2 & gefreut. \\
\hline 6) & ${ }^{d n i n}-\mathrm{gir}_{2}-\mathrm{su}-\mathrm{ke}_{4}$ & Ningirsu \\
\hline 7) & zapah-ni & hat (ihn) nach seiner Spanne \\
\hline 8) & mu-ni-ra & gemessen; \\
\hline 9) & {$[k u] s_{3}-5-a m_{6}$} & 5 Ellen lang \\
\hline 10) & kušs $-n i$ & hat er (ihn) nach seiner Elle \\
\hline 11) & mu-ni-ra & gemessen. \\
\hline 12) & kušs-5-zapab-1 & (Insgesamt:) 5 Ellen (und) 1 Spanne. ${ }^{23}$ \\
\hline 13) & dnin-gir - su-ke $_{4}$ & Ningirsu \\
\hline 14) & nam-gal-bul ${ }_{2}$-da & hat ihm aus großer Freude \\
\hline 15) & [nam-lug]al- & das Königtum \\
\hline 16) & [lagaši] & über Lagaš \\
\hline 17) & [mu-na-sum] & verliehen. \\
\hline
\end{tabular}

Der König E'annatum ist also vom obersten Stadtgott von Girsu, Ningirsu, gezeugt, von Inanna (von Uruk) mit einem Namen - dem Thronnamen, wie wir später sehen werden - benannt, wobei dieser Namensgebung eine gelehrte Etymologie zugrundegelegt wird: Der verkürzte Name $e_{2}$-an-na-tum, wörtlich »(Der) (ins) E'anna gebracht(e)«, wird als »Ins E'anna der Inanna von Ibgal habe ich(?) (ihn) gebracht ${ }^{24}$ oder auch »Für das E'anna der Inanna von Ibgal ist er geeignet $\aleph^{25}$ gedeutet. Anschließend setzt ihn Inanna in einer

23 Auf der Basis des spateren Maßstabs des Gudea von 1 Elle $=33 \mathrm{~cm}$ (s. dazu A. Salonen, 1965, Die Hausgeratte der alten Mesopotamier I, S. 277f.) ergibt dies $1,82 \mathrm{~m}$. 
Art Adoptionsritus ${ }^{26}$ der Göttin Ninhursag auf / in den Schoß, die ihn ihrerseits an ihrer Brust nährt. Der Vater Ningirsu äußert seine Freude über seinen Sprößling und mißt schließlich seine Körpergröße ab.

Viel deutlicher kann die Vorstellung einer Gotteskindschaft als Begründung für herrscherliche Legitimation kaum ausgedrückt werden, zumal daran nicht nur der Stadtgott, sondern auch zwei weitere höchste Gottheiten des sumerischen Pantheons (Inanna von Uruk und Ninhursag von Keš, die als Gemahlin des Enlil galt) beteiligt sind; es fehlt lediglich die direkte Erwähnung des obersten Gottes Enlil (von Nippur), doch dieser ist über die Vorstellung des Gottes Ningirsu als »Kriegsführer des Enlil «, ${ }^{27}$ die diesem Kontext vorausgeht, einbezogen. Darüber hinaus ist auf das gleich zweimalige Vorkommen des Begriffes zi »recht(mäßig), legitim« hinzuweisen, der den eigentlichen Adoptionsritus kennzeichnet: Es sind der Schoß und die Brust der Göttin Ninhursag (Kol. iv $25+27$ ), die den Herrscher legitimieren. Dem auf diese Weise legitimierten Sohn hat Ningirsu, der Stadtgott, »aus großer Freude das Königtum über Lagaš verliehen« (Kol. v 14-17).

Schließlich erklärt E'annatum (in Form eines Schlachtenrufs) ${ }^{28}$ seine Bereitschaft, die anstehende Aufgabe zu übernehmen, und daraufhin wird sein Thronname offiziell festgestellt. ${ }^{29}$ Die Einleitung zur Angriffserklärung gegen Umma zeigt deutlich, daß E'annatum mit Macht und Autorität ausgestattet und namentlich bestimmt ist vom obersten Stadtgott: Das hier bereits zum zweiten Male $\mathrm{M}^{30}$ verwendete Epitheton für den Herrscher $\mathrm{a}_{2}$-tuku(-e) »der Mächtige« zieht sozusagen die Summe aus den vorherigen Vorgängen um die Gotteskindschaft, und mit mu-pa ${ }_{3}$-da-dnin-gir ${ }_{2}$-su-ka(- $\left.\mathrm{ke}_{4}\right)$ »mit Namen bestimmt von Ningirsu « wird der Herrscher für die anstehende Aufgabe namentlich autorisiert. Hier liegt der Angelpunkt für die Ausformung der reichhaltigen Epitheta-Ketten, die erstmals bei diesem Herrscher festzustellen sind. Gleichzeitig wird auch der Unterschied deutlich zwischen der Vergabe des Thronnamens ( $=$ mu--sa ${ }_{4} » d e n$ Namen nennen «) durch Inanna und der namentlichen Bestimmung ( $=$ mu--pa $a_{3}$ »en Namen bestimmen «) für eine fest umrissene Aufgabe (= Autorisation) durch den Stadtgott.

E'annatum, derartig legitimiert und autorisiert, bringt seine staatspolitische Aufgabe zu einem erfolgreichen Abschluß und besiegelt das Ergebnis, nämlich die Wiedergewinnung des Territoriums des Gu'edenna für den Eigentümer, den Stadtgott Ningirsu, ${ }^{31}$ mit einem Vertrag, der bei den obersten Göt-

G.J. Selz (1998), S. 322-323 [43]-[44] bezeichnet diesen Vorgang als »Ankindungsritual«, das er den *Legitimationsritualen $\ll$ zurechnet.

27 [ur-s]ag-[den]-lil ${ }_{2}$-[la 2 ] in Kol. iv (4-) 5.

28 kur a-ne-se 3 »Das Fremdland für ihn (= Ningirsu)! $\ll$ in Kol. $~ 20$.

29 mu mu-ni-[gar(?)] wer (= Ningirsu(?)) hat dabei den Namen (fest)gesetzt« in Kol. v 29.

30 Beachte, daB E'annatum bereits bei seiner Bereitschaftserklärung so vorgestellt wurde (Kol. v 20-21).

31 Die von Kol. xiv 1 - xvi 7 reichende Aufzählung der einzelnen Feldernamen des Gu'edenna schlieBt mit der Feststellung, da $₫$ »'annatum, mit Namen bestimmt von Ningirsu, (diese Felder) in seine (= Ningirsus) Hand zurückgegeben hat« (Kol. xvi $8-11)$. 
tern des sumerisch-semitischen Pantheons beeidet wird. ${ }^{32}$ Nach dem Vertragsabschluß stellt sich E'annatum schließlich in einer umfassenden Epitheta-Kette vor, die die Legitimation seiner Herrschaft unter verschiedenen theologischen Aspekten betont:

E'annatum 1 Rs. v 42 - vi 9:

\begin{tabular}{|c|c|c|c|}
\hline S. v & $\begin{array}{l}\text { 50) } \\
51) \\
52) \\
53) \\
54) \\
55) \\
56) \\
\text { 1) } \\
\text { 2) } \\
\text { 3) } \\
4) \\
\text { 5) } \\
6) \\
\text { 7) } \\
8) \\
\text { 9) }\end{array}$ & 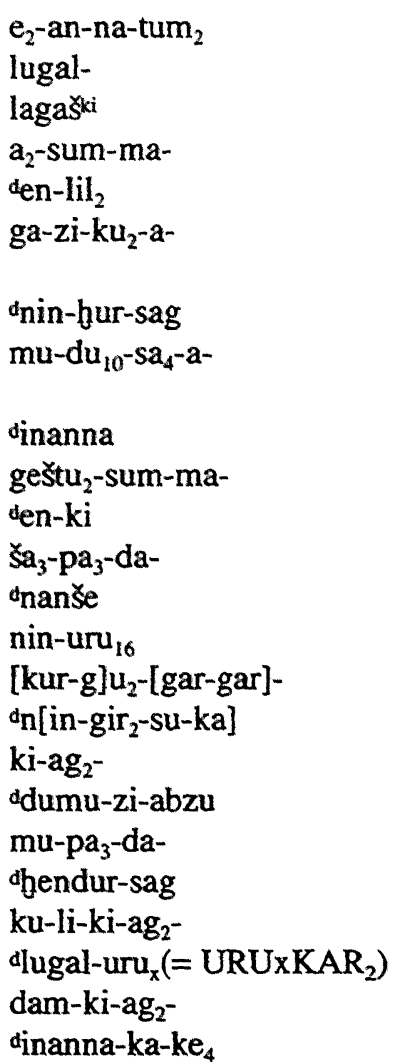 & $\begin{array}{l}\text { E'annatum, } \\
\text { der König } \\
\text { von Lagaš, } \\
\text { dem Kraft / Macht verliehen wurde } \\
\text { von Enlil, } \\
\text { der mit recht(mäßig)er Milch } \\
\text { genährt wurde } \\
\text { von Ninbursag, } \\
\text { dem ein guter Name genannt } \\
\text { wurde } \\
\text { von Inanna, } \\
\text { dem Weisheit verliehen wurde } \\
\text { von Enki, } \\
\text { der (im) Herzen erwählt wurde } \\
\text { von Nanš, } \\
\text { der gewaltigen Herrin, } \\
\text { der Fremdlandunterwerfer } \\
\text { des Ningirsu, } \\
\text { der Liebling } \\
\text { von Dumuziabzu, } \\
\text { der mit Namen bestimmt wurde } \\
\text { von Hendursag, } \\
\text { der geliebte >Freund } \\
\text { des Lugaluru, } \\
\text { des geliebten Gemahls } \\
\text { der Inanna. }\end{array}$ \\
\hline
\end{tabular}

32 E'annatum 1 xvi 12 - Rs. v 41: Die Eide werden geleistet auf

Enlil von Nippur,

Ninbursag von Kex, die Gemahlin Enlils,

Enki von Eridu,

den Mondgott Su'en von Ur,

den Sonnengott Utu von Larsa und

die Unterweltsgöttin Ereski<gab.

Vgl. dazu D.O. Edzard (1975), "Zum sumerischen Bid\%, in: AS 20, S. 64-68; H. Steible (1982), II, S. 49f.; G. Steiner (1986), „Der Grenzvertrag zwischen Lagas und Umma*, in: ASJ 8, S. 219ff. und jetzt G.J. Selz (1995), S. 119 [4], 126 [2], 223 [18), 275 [2], 286 [3]. 
Die ersten drei Epitheta sind schon behandelt. Neu ist die Weisheit, die dem Herrscher von Enki verliehen wird, neu ist die Erwählung durch Nanše, die Schwester des Stadtgottes Ningirsu, von der sich die I. Dynastie von Lagaš über den Namen des Dynastiegründers ur-dnanše (wörtlich: »Der (von) Nanše«) herleitet. Während die Bezeichnung »Fremdlandunterwerfer ${ }^{33}$ des Ningirsu « Rs. v 46 - vi 1 wiederum Bekanntes zusammenfaßt, sind auch der Bezug auf die Göttin Dumuzi'abzu und die namentliche Bestimmung durch den Gott Hendursag neu; diese beiden Gottheiten gehören in den Kreis von

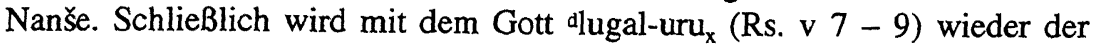
Bezug zu Inanna hergestellt.

Wir haben also einen klar strukturierten theologischen Aufbau der Legitimation vor uns, die einerseits die zentralen Gottheiten des sumerischen Pantheons (Enlil, Ninhursag, Inanna und Enki) einbindet, andererseits lokal wichtige Gottheiten (an der Spitze: Nanše und Ningirsu) berücksichtigt. Der Angelpunkt ist hier Enki, aus dessen Kreis die Göttin Nanše stammt.

Diese Epitheta-Kette bildet die Einleitung für einen historischen Tätigkeitsbericht, der mit der Aufstellung dieser Grenzstele endet. An dieser Stelle taucht nun überraschend im Stelen-Namen »Ningirsu, der Herr, (der) die Krone für Lumma (hat), ist das Leben des Pirigedenna-Kanals $\aleph^{34}$ der Name Lumma auf, von dem wir aus einem anderen Text des E'annatum (= E'annatum 2) wissen, daß er ein Parallel-Name des Herrschers selbst ist. In diesem Text stellt sich E'annatum in fast identischen Epitheta vor wie auf der Geierstele:

E'annatum 2 i 2 - iii 3:

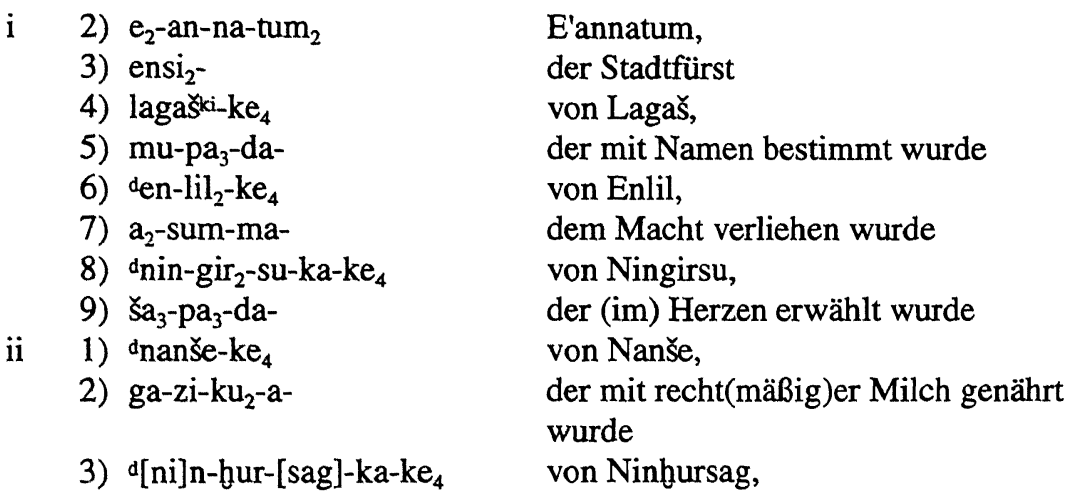

33 [kur-g] $\mathrm{u}_{2}$-[gar-gar], wörtlich: »(der) das Fremdland ganz und gar unterworfen hat mit dem Stadtgott Ningirsu gebraucht, s. die Zusammenschau der Belege bei $\mathrm{H}$. Behrens/H. Steible (1983), Glossar, S. 151 s.v. gu 2 --gar 2.; dazu jetzt auch G.J. Selz (1995), S. 224 [22] mit Anm. 1081.

34 d nin-gir $_{2}$-su / en men-lum-ma / nam-ti- / $\mathrm{i}_{7}$-pirig-eden-na, in Rs. $\times 26-29$; Übersetzung in Anlehnung an G.J. Selz (1995), S. 173 [8], S. 223f. [19]. 
4) $\mathrm{mu}-\mathrm{du} \mathrm{u}_{10}-\mathrm{sa}_{4}-\mathrm{a}-$

5) dinanna-ka-ke

6) geštu ${ }_{2}$-sum-ma-

7) den-ki-ka-ke 4

8) $\mathrm{ki}-\mathrm{ag}_{2}-$

9) ddumu-zi-abzu-ka-ke

10) giskim-ti-

11) dhendur-sag-ka-ke ${ }_{4}$

12) ku-li-ki-ag -

13)dlugal-uru $\mathrm{x}_{\mathrm{x}}-\mathrm{ka}_{\mathrm{a}}-\mathrm{ke}_{4}$

iii 1) dumu-a-kur-gal

2) ensi ${ }_{2}$ -

3) lagaš $\left[\mathrm{ki}^{\left.-\mathrm{ke}_{4}\right]}\right.$ dem ein guter Name genannt wurde

von Inanna,

dem Weisheit verliehen wurde

von Enki,

geliebt

von Dumuzi'abzu,

der Beistand (erhält)

von Hendursag,

der geliebte >Freund

von Lugaluru,

der Sohn des Akurgal,

des Stadtfürsten

von Lagaš.

Neben der Variation in der Abfolge der Epitheta ist die Konstanz der Zuständigkeiten der Gottheiten Nanše, Ninhursag, Inanna, Enki, Dumuzi'abzu und Lugaluru festzuhalten, während Enlil hier für die namentliche Bestimmung des Herrschers verantwortlich ist, Ningirsu für die Ausstattung mit Macht, und Hendursag als Beistand bezeichnet wird. Interessant ist hier die Abfolge der Götter, wo die für Lagaš-Girsu - NINA / Sirara zuständigen Geschwister Ningirsu und Nanše direkt auf den obersten Gott Enlil folgen, noch vor Ninhursag, Inanna und Enki. Die Sonderstellung dieser beiden Gottheiten kann man möglicherweise dadurch erklären, daß E'annatum seinen Tätigkeitsbericht in dieser Inschrift damit beginnt, daß er »dem Ningirsu Girsu wiederhergestellt, ihm die Mauer von Uruku ${ }^{35}$ gebaut (und) Nanš NINA / Sirara gebaut « hat (Kol. iii 4 - 11).

$\mathrm{Zu}$ beachten ist hier ferner, daß E'annatum sich als »Sohn des Akurgal, des Stadtfürsten von Lagaš» bezeichnet. An dieser Stelle manifestiert sich das Bedürfnis, die Legitimation für die Herrschaft aus der dynastischen Abfolge herzuleiten. Für alle Herrscher der I. Dynastie von Lagaš ist dies eine verbindliche Tradition, die lediglich von dem letzten Herrscher, Uru'inimgina, nicht übernommen werden konnte, da er ein homo novus war. ${ }^{36}$ Auch die gleichzeitigen Herrscher der Dynastien von $\mathrm{Umma}^{37}$ und $\mathrm{Ur}^{38}$ zeigen dieses Bestreben nach dynastisch begründeter Legitimation.

uru-ku 3 »Heilige Stadt«, in Girsu gelegen, s. jetzt G.J. Selz (1995), S. 6 [17].

Siehe die Tabelle bei W. Heimpel (1992), S. $7 f$.

Die direkte Abfolge Vater-Sohn ist nachzuweisen bei Enakalle und Urlumma (in der Inschrift Urlumma 1), bei II und Gił̌akidu (in der Inschrift Gił̌akidu 1) und bei U.U und Lugalzagesi (in der Inschrift Lugalzagesi 1), s. dazu H. Steible (1982), II, S. 267f. und 315f.

Die Abfolge Vater-Sohn ist in den originalen Inschriften nur für Lugalkiginedudu und Lugalkisalsi bezeugt; beide tragen auch den Titel »König von Ur« (in der Inschrift Lugalkisalsi 1 bei H. Steible, 1982, II, S. 306). Noch in der sumerischen Königsliste aus der altbabylonischen Zeit wird diese Genealogie überliefert (s. Th. Jacobsen, 1939, S. 100). 
An diesen Passus schließt sich an die Schilderung von drei verschiedenen Feldzügen, ${ }^{39}$ auf die dann der eigentliche Anlaß dieses Textes folgt. Hier findet sich erneut der Name des Lumma:

E'annatum 2 v 9-19:

\begin{tabular}{|c|c|}
\hline 9) & $\mathrm{u}_{4}-\mathrm{ba}$ \\
\hline 10) & $e_{2}$-an-na-tum $m_{2}-m^{40}$ \\
\hline 11) & $e_{2}$-an-na-tum ${ }_{2}$ \\
\hline 12) & $\mathrm{mu}-\mathrm{u}_{2}$-rum-m[a]-ni \\
\hline 13) & mu-tidnum-ni \\
\hline 14) & $1[u] m-m a-a^{41}$ \\
\hline 15) & ${ }^{d}$ nin-g $\left[i_{2}\right]-s u-[r] a$ \\
\hline 16) & a-gibil \\
\hline 17) & mu-na-dun \\
\hline 18) & lum-ma-gim-du ${ }_{10}$ \\
\hline 19) & mu mu-na- \\
\hline
\end{tabular}

\author{
Damals \\ hat E'annatum, \\ dessen eigener Name \\ E'annatum, \\ dessen Tidnum-Name \\ Lumma (ist), \\ dem Ningirsu \\ einen neuen Kanal \\ gegraben \\ (und) ihm (= Ningirsu) (zu Ehren) \\ Lummagimdu \\ (als) Namen genannt.
}

Hieraus geht klar hervor, daß E'annatum zwei Namen trägt, wobei der Name $\mathrm{e}_{2}$-an-na-tum ${ }_{2}$ der sumerische Thronname ist, während der Lumma-Name interessanterweise mit den semitischen Amurritern verbunden werden kann. ${ }^{42}$ Er wird hier gewiß aufgegriffen, weil es um die Anlage des nach ihm benannten lum-ma-gim-du ${ }_{10}$-Kanals geht. Dieser Lumma-Name findet sich auch noch in der Bezeichnung des Territoriums ${ }^{43}$ bzw. Kanals ${ }^{44}$ lum-ma-gir ${ }_{2}$-nunta- $\mathrm{Sa}_{3}-\mathrm{ku}_{3}$-ge-pa -da auf der (Grenz-)Stele der Inschrift E'annatum 60 ii 12: Über die Deutung dieses Namens als »Lumma vom >Hohen Weg< ins reine Innere berufen « hat G.J. Selz ${ }^{45}$ wahrscheinlich gemacht, daß lum-ma der Name dieses Herrschers war, ehe er mit einem anderen Namen inthronisiert wurde; darauf wird in der Geierstele zurückgegriffen. Der hier vorliegende

39 Der erste gegen Osten in die Gegend von Elam (Kol. iii 12 - iv 5), der zweite nach Süden (mit Uruk und Ur) und Südosten (bis Misime und Aru'a) (Kol. iv 6-24) und der dritte in Richtung Nordbabylonien gegen Aksak (Kol. iv 25 - v 8), s. dazu Tafel III.

$40 e_{2}$-an-na-tum ${ }_{2}$-ma hat G.J. Selz (1995), S. 171f. s.v. lum-ma [3] als "Etymologieversuch des Namens E'annatum* angesehen und hat Kol. v 10-12 mit »Der ins E'anna hineingebracht wurde<-E'annatum (ist) sein eigener Name« wiedergegeben.

41 lum-ma-a wird hier nach wie vor als Vokalharmonie für <*lum.a-e verstanden, wobei /e/ den Ergativ realisiert. Demgegenüber hat G.J. Selz (1995), S. 172 s.v. lum-ma [3] mit Anm. 753 lum-ma-a als regenslose Genitivverbindung ( $(D e r$ des Lumma«) aufgefaßt. Diese interessante Deutung läßt allerdings unberücksichtigt, daß man am Ende von lum-ma-a die Kennzeichnung der Subjektsposition als Ergativ erwartet, die dann nach dem Verständnis von G.J. Selz *lum-ma-ke 4 lauten müßte.

$42 \mathrm{Zu}$ Tid(a)num $=$ Amurru(m) s. jetzt D.O. Edzard, Reallexikon der Assyriologie und vorderasiatischen Archäologie, 7, Berlin 1987-1990, S. 438ff. s.v. Martu (Mardu).

43 So H. Steible (1982), I, S. 170 f.

44 So G.J. Selz (1995), S. 173 s.v. lum-ma [6].

45 G.J. Selz (1995), S. 173 s.v. lum-ma [6]. 
Kontext gibt gleichzeitig einen Einblick in den Vorgang der Erwählung, nämlich von dem $>$ Hohen Weg< (= Prozessionsstraße) ${ }^{46}$ weg ins Innere des Heiligtums. Ein vergleichbarer Vorgang wird unter En-te:me-na, dem zweiten Nachfolger des E'annatum, wie folgt beschrieben:

En-te:me-na 32 i $2 "-8 "$ :

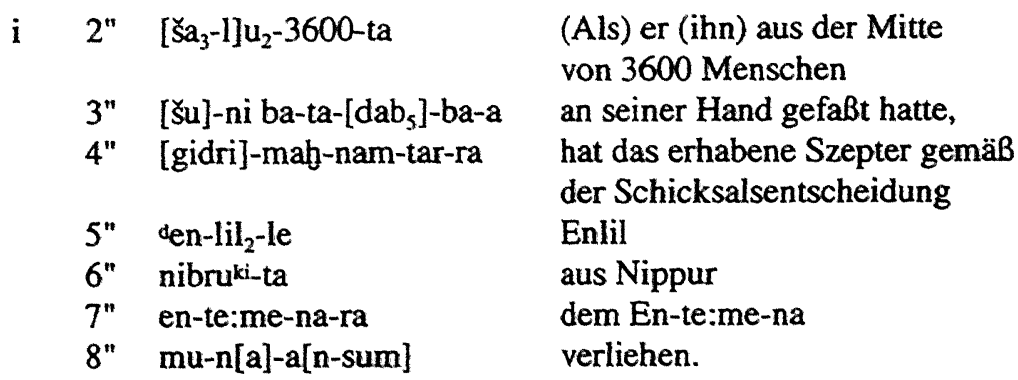

Die hier zusätzlich zu beobachtende Verleihung des Szepters durch Enlil an den Herrscher ist auch schon für E'annatum nachzuweisen, wie das bislang singuläre Epitheton für diesen Herrscher gidri-s[um-ma]-đnin-gir ${ }_{2}$-su-ka »dem das Szepter verliehen wurde von Ningirsu火 in E'annatum 69 iii 7-8 zeigt. Ergänzend zur Szepterverleihung durch Enlil erfahren wir aus der Inschrift En-te:me-na 26: 13-18, ${ }^{47}$ daß Nanše ihm das Königtum über Lagaš verliehen, und Ningirsu ihn dabei namentlich erwählt hatte $\left(=\mathrm{mu}-\mathrm{pa}_{3}\right)$.

Doch kehren wir zurück zu dem Text E'annatum 2: Für die politischen Implikationen der göttlichen Legitimation ist der unmittelbar folgende Passus in E'annatum 2 v 20 - vi 5 von großer Bedeutung:

$\begin{array}{lll}\mathrm{v} & 20 & \mathrm{e}_{2} \text {-an-na-tum } \\ 21 & \text { lu }_{2} \text {-inim-ma-se } \\ & \\ & \\ 22 & \text { ga- } \\ 23 & \text { enin-gir }_{2} \text {-su-ka-na-tum } \\ 24 & \text { ensi }_{2}- \\ 25 & \text { [lag]ašlid-ra } \\ 26 & \text { dinanna-ke } \\ \end{array}$

(Dem) E'annatum, der auf den Auftrag/Befehl angesetzt wurde von Ningirsu, E'annatum, dem Stadtfürsten von Lagaš, hat Inanna,

46 H. Steible (1982), II, S. 28.

47 En-te:me-na 26

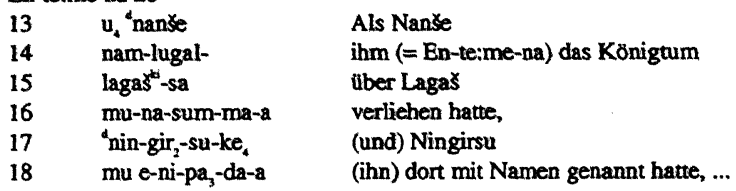


vi 1 ki an-na- $\mathrm{ag}_{2}-\mathrm{ga}_{2}$-da

2 nam-ensi ${ }_{2}-$

3 lagaški-ta

4 na[m]-lugal-kǐsi

5 mu-na-ta-sum die ihm (ihre) Liebe erweisen wollte, über das Stadtfürstentum von Lagaš hinaus das Königtum von Kiš verliehen.

Hier verleiht Inanna aus Liebe dem Herrscher über das Stadtfürstentum, das er schon besitzt, hinaus das »Königtum von Kiš «. ${ }^{48}$ Die Inanna, die das »Königtum von Kiš« verleiht, kann nicht die Inanna von Uruk sein, die ihn nach Ausweis der Geierstele in das gleichnamige E'anna von Ibgal in Lagaš gebracht hat, ${ }^{49}$ sondern muß die semitische Ištar der nordbabylonischen Stadt Kiš sein, ${ }^{50}$ in die - nach Auffassung der späteren Sumerischen Königsliste das Königtum nach der Sintflut vom Himmel herabgestiegen ist. Die beiden Göttergestalten Inanna - Ištar stehen hier für zwei Kulturbereiche, den sumerischen des Südens und den semitischen des Nordens - und für beide erklärt sich der Herrscher als legitimiert. Hintergrund dafür ist wiederum eine fest umrissene politische Aufgabe, die ihm von Ningirsu übertragen und in dem Epitheton lu $\mathrm{lu}_{2}$-inim-ma-se $\mathrm{S}_{3}$-ga-dnin-gir ${ }_{2}$-su-ka »der Mann, der auf den Auftrag/Befehl angesetzt wurde von Ningirsu $\aleph^{51}$ festgehalten wird, mit dem E'annatum sich explizit in diesem Passus vorstellt (Kol. v 21-22). Dieser Auftrag bestand in der Unterwerfung des Raumes östlich von Lagaక̌-Girsu, des nördlichen Gebietes von Kiš und Akšak bis hin zu dem weit entfernten Mari im Westen am mittleren Euphrat. ${ }^{52}$ E'annatum bringt ihn zu einem erfolgreichen Ende, wie aus E'annatum 2 vi 6 - vii 5 hervorgeht:

$$
\begin{aligned}
& \text { vi } 6 \mathrm{e}_{2} \text {-an-na-tum }{ }_{2} \text {-da } \\
& 7 \text { elam sag! e-da }-\mathrm{sig}_{3} \\
& 8 \text { elam kur-ra-na bi-gi } 4 \\
& \left.9 \text { kiß̌ki }[\mathrm{sag}] \text { e-d[a }] \mathrm{a}_{5}\right]-\mathrm{sig}_{3} \\
& 10 \text { lugal-akšak }{ }^{\mathbf{i}} \\
& 11 \text { kur-ra-na! }(=\mathrm{KI}) \text { bi-gi }{ }_{4} \\
& 12 e_{2} \text {-an-na-tum }{ }_{2} \\
& 13 \text { ensi }_{2} \text { - } \\
& 14 \text { laga }
\end{aligned}
$$

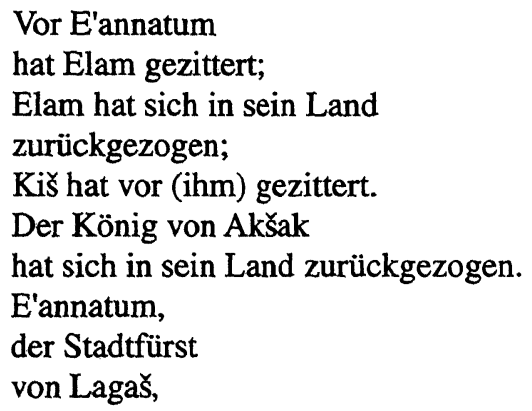

Vor E'annatum hat Elam gezittert; Elam hat sich in sein Land zurückgezogen; Kiš hat vor (ihm) gezittert. Der König von Akšak hat sich in sein Land zurückgezogen. E'annatum, der Stadtfürst von Lagaš,

48 Ansonsten wird das »Königtum von Lagaš ( $=$ nam-lugal-laga ${ }^{\nless k i}$ ) von dem Gott Lugaluru an Enannatum 1. (Enannatum 19 ii 8 - iii 1) und von Nanše an En-te:me-na (En-te:me-na 26: 13-18) verliehen.

49 Zu Lage des Ibgal in Lagaš s. G.J. Selz (1995), S. 148 s.v. dinanna(-k) [8].

50 Vgl. dazu schon S. Franke (1995), Königsinschriften und Königsideologie, S. 55 mit Anm. 88 und 89.

51 Die in diesem Epitheton nachzuweisende Gottheit ist immer gleichzeitig Adressat der jeweiligen Inschrift: In E'annnatum 2 v $21-22$, vii $15-16 ; 5$ ii 8 - iii $1 ; 8$ ii' $3^{\prime}-4$ ' und 11 vi $6-7$ ist dies Ningirsu, in Enannatum I 35 iii 7-8 Inanna (von) Ibgal und in En-te:me-na 79 vi 8-9 Lugalemus.

Siehe dazu Tafel III. 
15 kur-gu $_{2}$-gar-gar-

16 dnin-gir $_{2}$-su!-ka-ke

17 elam subarki

18 urua

19 a-subur-ta

$20 \mathrm{aga}_{3}-\mathrm{kar}_{2} \mathrm{bi} \mathrm{-se}$

21 kišsi akšak $^{\mathbf{k i}}$

$22 \mathrm{ma} \mathrm{ri}_{2}$ !ki

23 an-ta-sur-ra-

vii 1 dnin-gir ${ }_{2}$-su-ka-ta

$2 \mathrm{aga}_{3}-\mathrm{kar}_{2} \mathrm{bi}-\mathrm{s}\left[\mathrm{e}_{3}\right]$

3 dnin-gir ${ }_{2}$-su-ra

4 lum-ma-gim-du 10

5 mu-na-us der Fremdlandunterwerfer

des Ningirsu,

hat Elam, Subar

(und) Arawa ${ }^{53}$

(ausgehend) vom Asuhur(-Kanal)

mit Waffen geschlagen.

Kiš, Akšak

(und) Mari

hat er (ausgehend) vom Antasurra

des Ningirsu

mit Waffen geschlagen

(und) hat dem Ningirsu

den Lummagimdu(-Kanal)

angelegt(?).

Diese Passage zeigt, daß hier eine militärische Operation nach ihrem erfolgreichen AbschluB über einen göttlichen Auftrag sanktioniert und eine international anerkannte Legitimation gesucht und proklamiert wird, d.h. die Legitimation erfolgt - wie auch in der Geierstele - auf Grund einer erbrachten Leistung, ist also eine Legitimation ex eventu. Die Anlage des Kanals lumma-gim-du ${ }_{10}$ verrät den eigentlichen Grund dieser Unternehmung; denn mit der Wasserzufuhr vom Euphrat her wurde das Territorium des Stadtstaates Lagaš-Girsu endlich unabhängig von der durch Umma in der nördlichen Nachbarschaft immer wieder gefährdeten Wasserversorgung vom Tigris her. So erklärt sich problemlos die Wahl der semitischen Komponente im Kanalnamen, nämlich lum-ma, ${ }^{54}$ mit der der sumerische Herrscher E'annatum aus dem südbabylonischen Lagaš Rücksicht nimmt auf die semitische Bevölkerung in den betroffenen Gegenden Nordbabyloniens.

Eine Widerspiegelung dieser Vorstellungen findet sich noch bei dem letzten Herrscher dieser I. Dynastie von Lagaš, Uru'inimgina. Interessanterweise sind es hier weder Inanna, noch Enlil, noch Nanše, die ihm das Königtum verleihen, sondern der Stadtgott seiner Residenz, Ningirsu, wie dem E'annatum (vgl. E'annatum 1 v 13-17). Der politisch wie militärisch bedrängten Lage des Uru'inimgina entspricht das Epitheton für Ningirsu, nämlich ur-sagden- $-\mathrm{lil}_{2}-\mathrm{la}_{2}-\mathrm{ke}_{4}$ »Kriegsführer des Enlil«. Ningirsu ist es auch, der wie den Ente:me-na, seinen Vorvorgänger, den Uru'inimgina erwählt, indem er ihn an der Hand faßt:

53 Zu den Schreibungen und der Lage von urua = Arawa s. ausfuhrlich P. Steinkeller (1982), wThe question of Marbasi «, in: ZA 72, S. 244ff. mit Anm. 26; dazu jetzt auch G.J. Selz (1991), „Elam und Sumer«, in: CRRA 36 (1989) S. 27-29, bes. S. 28 mit Anm. 10. 
Uru'inimgina 4 vii 29 - viii 13:

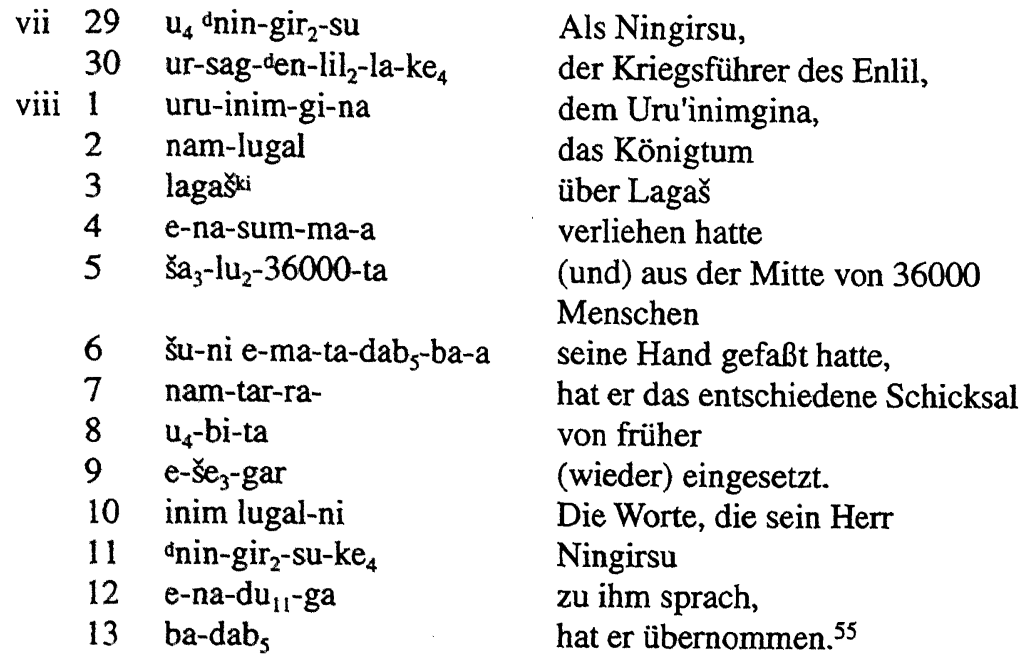

Diese Erwählung erfolgt in einem Kontext, in dem der Herrscher die Legitimation für seine Restaurationsregelungen braucht, die als wortwörtliche Gottesoffenbarung dargestellt werden. Diese Neuordnung hat das Ziel, die alten Verhältnisse wiederherzustellen, unter denen das Stadtgötterpaar mit Familie alleiniger Eigentümer von Grund und Boden des Stadtstaates war; dieses Prinzip war unter den Vorgängern des Uru'inimgina, vor allem wohl unter En-te:me-na - wenn wir diesen Namen richtig als en-me-te-na »Herr durch sich selbst ${ }^{56}$ umdeuten - in zunehmendem Maße, wohl unter semitischem Einfluß, durchlöchert worden, - ein Prinzip, das Uru'inimgina am Ende der Darstellung der Mißstände mit dem semitischen Fremdwort be ${ }_{6}-\mathrm{lu}_{5^{-}}$ da »Herrschaftsnormen $\ll, ~ » H e r r s c h a f t s a n s p r u c h ~ «$ charakterisiert. ${ }^{57}$

Noch 200 Jahre später wird ein Herrscher der II. Dynastie von Lagaš, nämlich Gudea, mit einem fast identischen Wortlaut seine Legitimation begründen:

55 Siehe zu diesem Kontext jetzt auch G.J. Selz (1998), S. 324. Vgl. schon das Epitheton für En-te:me-na in En-te:me-na. 28 v $30=29$ vi $11-12 \mathrm{lu}_{2}$ inim-dingir-re 2 -ne ( $)$ dabs-ba $» d e r$ Mann, der den Auftrag übernommen hat*.

56 Vgl. schon die Übersetzung »his own lord« bei B. Alster (1974), »En.mete.na >His own Lord«, in: JCS 26, S. 178-180.

57 Vgl. Uru 'nimgina 4 iii $2-$ vii $28=5$ iii 3 vii 11: "Seit fernen Tagen, seit der Same aufgegangen ist, zu dieser Zeit waren...$<\left(=\right.$ Uru'inimgina 4 iii 5 - vii $25=5$ iii 6 - vii 8 ) die Herrschaftsnormen $\left(=b_{6}{ }^{-l_{5}}\right.$ da), die seit diesen Tagen galtenж; s. dazu G.J. Selz (1998), S. 323-324 [45] und jetzt auch H. Steible (1999), „Zu den Nahtstellen in den altmesopotamischen Codices«, in: Joachim Marzahn/Hans Neumann (Hg.), Assyriologica et Semitica. 
Gudea Statue B iii 6-11:

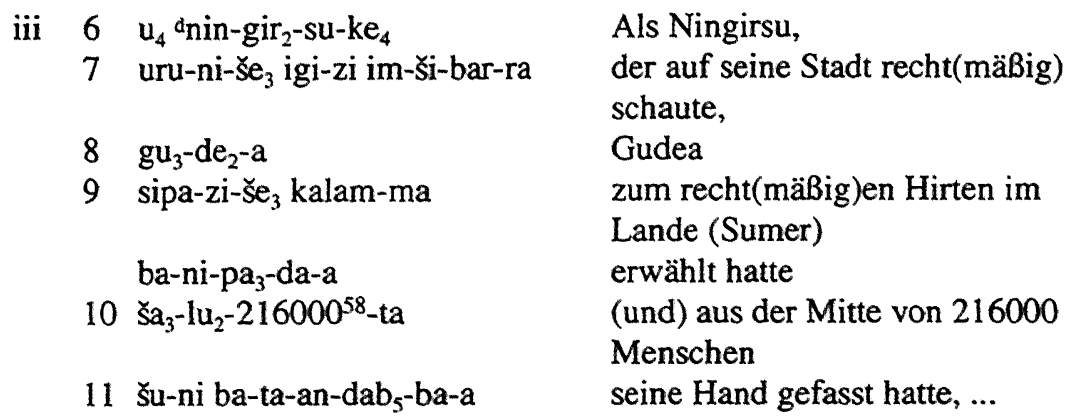

Der bewußte Rückgriff auf Formulierungen des E'annatum bzw. En-te:me-na ist bei Gudea auch zu beobachten in den Epitheta, mit denen er seine Verbindungen zu den wichtigsten Gottheiten des lokalen Pantheons von Lagaš-Girsu etwa in Statue B bekundet:

Gudea Statue B ii 8 - iii 5:

ii 8 sipa-ša $a_{3}$-ge-pa $a_{3}$-da-

9 dnin-gir 2 -su-ka-ke

10 igi-zi-bar!-ra-

11 inanše-ke ${ }_{4}$

12 a $a_{2}$-sum-ma-

13 dnin-DAR-a-ke ${ }_{4}$

$14 \mathrm{lu}_{2}$-inim-ma-se ${ }_{3}$-ga-

15 dba-ba $6-\mathrm{ke}_{4}$

16 dumu-tu-da-

$17 \mathrm{dga}_{2}-\mathrm{tum}_{3}-\mathrm{du}_{10}-\mathrm{ke}_{4}$

18 nam-nir-gal 2 -gidri-mah-

sum-ma-

19 dig-alim-ka-ke

iii 1 zi-ša - gal $_{2}$-la-šu-dagal$\mathrm{du}_{11}-\mathrm{ga}$

2 dšul-ša $a_{3}-g a-k a-k e_{4}$

3 sag-zi-ukkin-na-

pa- $\mathrm{e}_{3}-\mathrm{a}$
Der Hirte, der im Herzen erwählt wurde von Ningirsu, der recht(mäßig) angeschaut wurde von Nanše, dem Kraft verliehen wurde von Nin-DAR-a, der Mann, der auf den Befehl angesetzt wurde von Baba, das leibliche Kind der Gatumdu, dem Ansehen (und) das erhabene Szepter verliehen wurde von Igalim, der mit Lebensodem reichlich versehen wurde von Šulšaga, der (als) das recht(mäßig)e Haupt der Ratsversammlung sichtbar gemacht wurde 
Einbezogen sind hier die Götterpaare von Girsu (Ningirsu und Baba) und NINA / Sirara (Nanše und Nin-DAR-a), die uralte nichtsumerische Stadtgottheit von Lagaš (Gatumdu), zwei Söhne aus der Verbindung des Götterpaares von Girsu (Igalim und Šulšaga) und schließlich Gudea's persönlicher (Schutz)gott Ningišzida.

Zwischen die I. und II. Dynastie von Lagaš schiebt sich der Aufschwung und Niedergang der Dynastie von Akkade ${ }^{59}$ mit der Schaffung des ersten Territorialstaates in Vorderasien. Sargon - šarru-kin »der König ist rechtmäBig / legitim « wie sein akkadischer Name lautet -, dem Begründer dieser Dynastie (ca. 2340-2284 v. Chr.), gelang es, ausgehend von Kiš nach dem entscheidenden Sieg über Lugalzagesi von Umma und Uruk ein Reich zu errichten, das sich vom Persischen Golf bis zum Mittelmeer erstreckte. Unter seinen Titeln šar (LUGAL) a-ka $\mathrm{Kae}_{3} \mathrm{KI}$ »König von Akkade«, šar matim (LUGAL.KALAM.MA.KI) »König des Landes (Sumer)« und šar (LUGAL) kiš $» \mathrm{König}$ von $\mathrm{Ki}{ }^{3} \aleph^{60}$ war der letztgenannte, in Anlehnung an die altsumerische Tradition, besonders prestigeträchtig, ${ }^{61}$ weil Sargon diesen Titel in zahlreichen Inschriften als einzigen trägt und seine beiden nachfolgenden Söhne Rimuš und Maništusu ausschließlich diesen Titel gebrauchen. ${ }^{62}$ Diese beiden waren im wesentlichen damit beschäftigt, das Reich ihres Vaters zu sichern. Erst Sargons Enkel Narāmsin (2260-2223 v. Chr.) war in der Lage, das Reich auf den assyrischen Norden auszudehnen und dort zu stabilisieren, wozu Maništusu bereits angesetzt hatte.

Diese politischen Erfolge spiegeln sich auch darin wider, daß die bisherigen Titel šar matim und šar (LUGAL) kiš aufgegeben werden zugunsten von da-num $m_{2} \gg$ der Mächtige « ${ }^{63}$ und lugal-an-ub-da-limmu ${ }_{2}$-ba = šar (LUGAL) kiib-ra-tim ar-ba-im »König der Vier Weltgegenden $\ll_{.}{ }^{64}$

59 Siehe Tafel 1.

60 Vgl. dazu S. Franke (1995), S. 94-95.

61 Diesen Titel trägt Sargon als einzigen in der Inschrift Sargon C 2, wo von 34 siegreich bestandenen Kämpfen vom Persischen Golf und entlang des Euphrat bis hin nach Ebla (unweit des heutigen Aleppo) berichtet wird. Indirekt ist auch auf den Sieg über Lugalzagesi von Uruk in den Beischriften (a) und (b) angespielt.

62 S. Franke (1995), S. 100. - Hinter der konsequenten Wiedergabe kiš ohne das Ortsdeterminativ KI ist häufig eine Schreibung für kiššatu vermutet worden, so daß šar kił als »König der Gesamtheit« zu verstehen wäre (s. dazu etwa P. Steinkeller, 1993, S. 120). Dabei sollte allerdings nicht übersehen werden, $\mathrm{da} B$ schon die sumerische Königsliste in der Stadt Kiš den Beginn des babylonischen Königtums nach der Flut festmacht (s. Th. Jacobsen, 1939, S. 76 i 40-42).

63 Die geläufige Entsprechung im Sumerischen ist kal-ga. Da kal-ga jedoch nie frei, sondern ausschließlich als attributives Adjektiv verwendet wird, fragt es sich, ob bei dem freien Gebrauch des akkadischen dannum »der Mächtige« Naramsin auf das ältere Epitheton $a_{2}$-tuku »der Mächtige« für E'annatum von Laga zurückgreift (s. oben S. 5). - Unter Naramsins Nachfolger Sarkalisarri wird dieses Epitheton zu Sarrum (LUGAL) dannum = lugal-kal-ga »der mächtige König« erweitert, s. die Belege bei B. Kienast/W. 
In allen originalen Inschriften des Narāmsin, mit Ausnahme einer einzigen aus Ur, ${ }^{65}$ wird der Herrscher, wenn er diesen letztgenannten Titel trägt, mit dem Gottesdeterminativ geschrieben, eine ungeheuerliche Neuerung in der keilinschriftlichen Überlieferung. Für diese Vergöttlichung des Herrschers ist wiederum die internationale Anerkennung auf Grund des Reichsbestandes, vor allem auch unter Einbeziehung des assyrischen Nordens, die Grundlage und Voraussetzung. In der Inschrift der Statue aus Bassetki, die genau in jenen nördlichen Gebieten des späteren Assyrien gefunden wurde, wird dies folgendermaßen dargestellt:

Narămsin 1 i 20 - iii 7:66

i 20 al ši in $p u-u \check{s}-q i_{2}-i m$

21 SUHUUS.SUHUS

22 URUKI-li2-su

$23 u-k i_{2}-n u$

24 URUKI-su

$25-$ ii 23 ...

ii $24 \quad i_{3}-l i_{2}$-is URUKI-su-nu

iii $1 \quad \mathrm{a}-\mathrm{ka}_{3}-\mathrm{de}_{3} \mathrm{KI}$

$2 i$-tar 2 -su-ni-is-<su>-

$3 m a$

$4 \quad q a b_{2}-l i$

$5 \quad \mathrm{a}-\mathrm{ka}_{3}-\mathrm{de}_{3}$

$6 \mathrm{E}_{2}-s u$

$7 \quad i b-n i-u_{3}$
Weil er (=Narāmsin) in d(ies)er Notlage die Fundamente

seiner Stadt

gefestigt hat, haben (die Bürger) seine( $r$ ) Stadt

(bei den Göttern) ... (= NN)

zum Gott ihrer Stadt

Akkade

ihn (= Narămsin) sich erbeten

und

inmitten von

Akkade

seinen Tempel

errichtet.

Diese vorgebliche Bitte der Bürger wird gerichtet an die zentralen Götter Süd- und Nordbabyloniens ${ }^{67}$ und des Gebietes am mittleren Euphrat (im heutigen NO-Syrien):

1. Inanna von E'anna (Uruk),

2. Enlil von Nippur,

3. Dagan von Tuttul (=Tell Bi'a),

4. Ninhursaga von Keš,

5. Enki von Eridu,

6. Suen (/Sin) von Ur,

Sommerfeld (1994), Glossar zu den altakkadischen Königsinschriften, in: FAOS 8, S. 147 s.v. lugal (b) 6'., 293 s.v. కarrum (b) 20 .

Siehe die Belege bei B. Kienast/W. Sommerfeld (1994), S. 293 s.v. \arrum (b) 22".

UET VIII 11 = Naramsin 10 bei I.J. Gelb/B. Kienast (1990), S. 81-82.

66 Bei I.J. Gelb/B. Kienast (1990), S. 81-82.

67 Siehe Tafel III. 
7. Utu (/ Šamaš) von Sippar und

8. Nergal von Kutha.

Hintergrund dieser Bitte ist die »Notlage « (= pušqum), die Narāmsin selbst so zusammenfaßt, daß »die vier Weltgegenden insgesamt gegen ihn rebellierten ${ }^{68}$ so $\mathrm{da} \beta$ »er in einem (einzigen) Jahr neun Schlachten bestehen « ${ }^{69}$ mußte. Er bestand sie siegreich »durch die Liebe, die Ištar ihm erwiesen hat $\ll ; 70$ man denkt bei dieser Formulierung unmittelbar zurück an E'annatum, dem die (Inanna /) Ištar der Stadt Kiš, »die ihm (ihre) Liebe erweisen wollte, ... das Königtum von Kiš verliehen hat «. ${ }^{71}$

In diesem einen Titel »König der Vier Weltgegenden« werden also zahlreiche Legitimationsvorstellungen zusammengefaßt. In dem Rang eines Stadtgottes ist Narāmsin nun auch Herr von Grund und Boden seiner Städte und damit seines Reiches und dadurch ex officio dazu berechtigt, bisheriges Tempelland für sich und seinen Palast zu beanspruchen. ${ }^{72}$ Damit erreicht die Entwicklung einen Höhepunkt, die in Nordbabylonien weit gestreut zu beobachten ist (vor allem in den Archiven von Mugdan): die Dominanz der königlichen Domänen und der Privathaushalte in den ökonomischen Institutionen, die vor dem Hintergrund des sich immer stärker herausbildenden privaten Landbesitzes zu sehen ist. ${ }^{73}$ Diese Entwicklung steht in vollem Gegensatz zu der Südbabyloniens mit den Tempeldomänen.

Dieser entscheidende Titel wird in akkadischer Fassung und seiner sumerischen Entsprechung lugal-an-ub-da-limmu ${ }_{2 / 5}-\mathrm{ba}^{74}$ von Šulgi, dem 2. König der III. Dynastie von Ur (ca. 2093-2046 v. Chr.) wieder aufgenommen, ${ }^{75}$ und zwar wiederum

1. in Verbindung mit der Schreibung des Gottesdeterminativs vor dem Herrschernamen und

2. auf Grund der Tatsache, daß er ein Reich besaß, das dem unter Naramsin vergleichbar war. Seine Nachfolger Amar-Sin und Šū-Sin halten an dieser Tradition fest.

Mit dem Ende der Ur-III-Zeit wird dieser Titel schließlich aufgegeben, und das Prestige der allseitig von Herrschern angestrebten Legitimation ist fortan

68 Kol. i 5-9.

69 Kol. i 13-15.

70 Kol. i 10-12.

71 E'annatum 2 v 26 - vi 5 (siehe S. 76-77).

72 Vgl. dazu schon B. Kienast (1973), „Der Weg zur Einheit Babyloniens« in: OrNS 42, S. 495-496.

73 Siehe dazu P. Steinkeller (1993), S. 122 mit Anm. 41.

74 Der sumerische Titel findet sich schon in den Inschriften Utubegal 1, 2, 3, 5 und 6, s. dazu H. Steible (1991), II, S. 324ff.

75 Dieser Titel ist in akkadischer Fassung seit dem 21. Regierungsjahr S̆lgi's nachzuweisen und in sumerischer Sprache seit dem 27. Jahr, s. dazu D. Frayne (1997), S. 92. 
bis in die Zeit des Hammurabi von Babylon hinein verbunden vor allem mit dem Standard-Titel aller fünf Könige der III. Dynastie von Ur, lugal-uri $2 / 5^{\mathbf{k}^{\mathrm{i}}-}$ ma $»$ König von $U r \ll .{ }^{76}$

Ein bisher beiseite gelassener Strang der Legitimation, der mit dem Titel en, und dadurch mit den frühdynastischen Herrschern von Uruk verbunden ist, muß an dieser Stelle noch aufgegriffen werden.

Es ist Inanna, die Stadtgöttin von Uruk, die diesen Titel verleiht, dessen Bildzeichen bis ans Ende des 4. Jahrtausends v. Chr. zurückreicht und sich mit dem »Becher-Tablett« verbinden läßt, das auf dem obersten Bildstreifen der Uruk-Vase dargestellt ist, innerhalb der Zone, die durch die Schilfbündel als Bereich der Inanna gekennzeichnet ist. ${ }^{77}$ Selbst wenn die schriftlichen Zeugnisse für die Vorstellung der »Heiligen Hochzeit« erst viel später einsetzen, kann man vermuten, daß auch in der frühdynastischen Periode ein analoger Ritus bestanden hat, auf Grund dessen der Herrscher eine ganz spezifische und für Uruk geltende Legitimation erhielt, die in der Erwählung zum Ehegatten der Stadtgöttin und damit zum »Herrn von Uruk « bestand, was eine Gleichsetzung mit ihrem mythischen Gemahl Dumuzi bedeutete. ${ }^{78}$

Lugalkiginedudu, ein Zeitgenosse des En-te:me-na von Lagaš, mit dem die bisher bekannte historische Abfolge der Herrscher von Uruk beginnt, stellt sich in der Weihinschrift 2 folgendermaßen vor:

Lugalkiginedudu 2: 1-14:

\begin{tabular}{|c|c|}
\hline 1 & den- $\mathrm{lil}_{2}$ \\
\hline 2 & lugal-kur-kur-ra \\
\hline 3 & lugal-ki-gin- $\mathrm{ne}_{2}-\mathrm{du}_{7}-\mathrm{du}_{7}$ \\
\hline 4 & $\mathrm{u}_{4}$ den-lil ${ }_{2}$-le \\
\hline 5 & $g_{3}-z i$ e-na-de ${ }_{2}-\mathbf{a}$ \\
\hline 6 & nam-en \\
\hline 7 & nam-lugal-da \\
\hline 8 & e-na-da-tab-ba-a \\
\hline 9 & unuki-ga \\
\hline 10 & nam-en \\
\hline 2 & mu-ak-ke ${ }_{4}$ \\
\hline 12 & uri $_{2}^{\text {ki-ma }}$ \\
\hline 13 & nam-lugal \\
\hline & mu-ak-ke ${ }_{4}$ \\
\hline
\end{tabular}

Enlil, der Herr aller Länder, läßt, als zu Lugalkiginedudu Enlil in recht(mäßig)er Weise gesprochen (und) ihm die Enschaft mit dem Königtum verbunden hatte, (ihn) in Uruk die Enschaft ausüben, (ihn) in Ur das Königtum ausüben. 
Enlil, der oberste Gott des sumerischen Pantheons, verleiht hier dem Herrscher zu der Enschaft von Uruk das Königtum, das auf die Stadt Ur spezifiziert wird.

In einer parallelen Weihinschrift ${ }^{79}$ ist es Inanna als Stadtgöttin von Uruk (/ E'anna), die diese Legitimation in der gleichen Weise vornimmt, wobei Lugalkiginedudu zusätzlich mit dem Prestige-Titel lugal-kiški »König von Kiš« versehen ist. Darin spiegelt sich offensichtlich eine analoge Situation wider, die für E'annatum von Lagaš die doppelte Legitimation durch die Inanna von Uruk und die Ištar von Kiš mit sich brachte. ${ }^{80} \mathrm{An}$, der Stadtgott von Uruk, steht in dieser Inschrift, und zwar mit dem Epitheton lugal-kur-kur-ra »der Herr aller Länder «, interessanterweise in der Position, die Enlil in der vorhergenannten einnimmt.

Der vorletzte Herrscher dieser Uruk-Dynastie, Enšakušanna, trägt die Titel en-ki-en-gi »Herr von Ki'engi« und lugal-kalam-ma »König des Landes (Sumer) $\ll^{81}$ Das darf wohl so verstanden werden, daß die Legitimation im ersten Titel sich auf die Stadt Uruk und die umliegende Region bezieht, während »König des Landes (Sumer) « auf Ur und dessen Umland zu beziehen ist.

Den Schlüssel zu dieser Regionalverteilung der Titel liefert eine Inschrift des zwischen beiden Königen einzuordnenden Herrschers Lugalkisalsi:

Lugalkisalsi 1: 5'-7':

5' lugal-kisal-si

6' lugal-unuki-ga-ke ${ }_{4}$

$7^{\prime} \quad$ lugal-uri ${ }_{2}^{\text {ki-ma- }} \mathrm{ke}_{4}$
Lugalkisalsi, der König von Uruk, der König von Ur, ...

Hier ist bemerkenswert, daß Lugalkisalsi nicht nur den alten Uruk-Titel en zugunsten von lugal aufgegeben hat, ${ }^{82}$ sondern auch gleichzeitig seine genealogische Verbindung zu seinem Vater und Vorgänger darstellt. ${ }^{83}$ Diese Verbindung zwischen einem genealogischen Bezug und dem Titel lugalunuki-ga »König von Uruk « ist dann auch bei dem letzten Herrscher von Uruk, Lugalzagesi, festzustellen:

\section{Lugalzagesi 1}

$$
\begin{array}{ll}
3 & \text { lugal-za } \\
4 & \text { luge-si } \\
5 & \text { lugal-unuki-gal-ga } \\
&
\end{array}
$$

Lugalzagesi,

König von Uruk,

König des Landes (Sumer)

79 Lugalkiginedudu 4.

80 Siehe oben S. 11f. zu E'annatum 2 v 20 - vi 5 mit Anm. 45.

81 Enšakuకanna 1:4-5.

82 Mit diesen beiden Titeln stellt sich Lugalkisalsi auch in der Bauinschrift Lugalkisalsi 5: 4-5 vor.

83 Lugalkisalsi 1: 1'-3', wo er sich «S[ohn ...] des Lugalkiginedudu, des Königs von Uruk, des Königs von Ur« nennt. 
$\ddot{9} \quad$ dumu- $U_{2} \cdot U_{2}$

10 [en]si ${ }_{2}$-ummaki
Sohn des U.U,

des Stadtfürsten (von) Umma, ... .

Aus Umma stammend, knüpft Lugalzagesi mit dem ersten Titel an Lugalkisalsi an und sucht mit dem zusätzlichen Titel lugal-kalam-ma »König des Landes (Sumer)« die Legitimation über seinen Amtsvorgänger Enšakušanna in Uruk. Aus dem en-unuki-ga »Herr von Uruk« ist nun ein lugal-unuki-ga »König von Uruk « geworden, der trotz seiner ortsfremden Herkunft mit seiner Genealogie in die Öffentlichkeit treten kann.

Zusammenfassend ergibt sich folgendes Spektrum der Legitimationsmöglichkeiten für Herrschaft im Alten Mesopotamien des 3. Jahrtausends v. Chr. $\mathrm{Zu}$ nennen sind

- die Gotteskindschaft und damit verbundene Ausstattung,

- die Erwählung mit Verleihung des Thronnamens und der Regalia,

- die Legitimation ex eventu, die auf Grund einer Autorisation zu einer bestimmten Aufgabe erreicht wird,

- die Erwählung zum Gemahl einer Stadtgöttin und

- der Nachweis einer genealogischen Abkunft.

Diese Legitimationen berechtigen zur Führung bestimmter Titel, wobei die

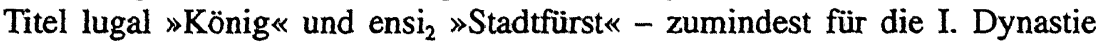
von Lagaš - zusammengesehen werden können, ${ }^{84}$ denen der lokal gebundene des en »Herr« gegenübersteht.

Die Zusammenschau des Materials zeigt, daß Genealogien nur zusätzlich bei den Titeln ensi ${ }_{2}$ und lugal auftreten, nie aber im Zusammenhang mit dem Titel en. Man kann sogar beobachten, daß der Titel en dann zugunsten von lugal aufgegeben wird, wenn das Bedürfnis nach dynastischer Legitimation besteht. Die Konsequenz aus den frühdynastischen Verhältnissen war die Vergöttlichung des Herrschers in der altakkadischen Zeit unter Naramsin, die vor dem Hintergrund des sich immer stärker herausbildenden privaten Landbesitzes der Überwindung der bisherigen Kleinstaaterei zugunsten eines Imperiums Rechnung trägt. Alle folgenden Herrscher und Dynastien in Mesopotamien - d.h. Babylonien und Assyrien - werden in der einen oder anderen Weise aus diesen vorgegebenen Modellen ihre Legitimation herleiten.

84 W. Heimpel (1992), S. 6 hat diese Feststellung so definiert, daß »sie Könige waren, die sich >Stadtfürsten « nanntenk. 


\section{Literatur}

Alster, Bent (1974): »En.mete.na: His own Lord«, in: Journal of Cuneiform Studies, 26, S. 178-180.

Behrens, Hermann/Steible, Horst (1983): Glossar zu den altsumerischen Bau- und Weihinschriften, in: FAOS 6, Wiesbaden.

Behrens, Hermann u.a. (Hg.)(1989): Dumu-e $e_{2}-d u b-b a-a$. Studies in Honor of Ake W. Sjöberg, in: Occasional Publications of the Samuel Noah Kramer Fund, 11, Philadelphia.

Biggs, Robert D. (1974): Inscriptions from Tell Abū Saläbikkh, in: Oriental Institute Publications, 99, Chicago/London.

Cooper, Jerrold S. (1983): Reconstructing History from Ancient Inscriptions: The Lagash-Umma Border Conflict, in: Sources from the Ancient Near East, 2/1, Malibu.

Cooper, Jerrold S. (1986): Sumerian and Akkadian Royal Inscriptions I. Presargonic Inscriptions, New Haven.

Dietrich, Manfried/Loretz, Oswald (Hg.)(1998): dubsar anta-men. Festschrift für Willem H. Ph. Römer zur Vollendung seines 70. Lebensjahres mit Beiträgen von Freunden, Schülern und Kollegen, in: AOAT 253, Münster.

Edzard, Dietz O. (1975): »Zum sumerischen Eid«, in: Assyriological Studies, 22, S. 63-98.

Edzard, Dietz O./Farber, Gertrud/Sollberger, Edmond (1977): Die Orts- und Gewässernamen der präsargonischen und sargonischen Zeit, in: Répertoire Géographique des Textes Cunéiformes, 1, Wiesbaden.

Franke, Sabina (1995): Königsinschriften und Königsideologie. Die Könige von Akkade zwischen Tradition und Neuerung, Münster.

Frayne, Douglas (1993): Sargonic and Gutian Periods (2334-2113 BC), in: RIME 2, Toronto.

Frayne, Douglas (1997): Ur III Period (2112-2004 BC), in: RIME 3/2, Toronto.

Gelb, Ignace J./Kienast, Burkart (1990): Die altakkadischen Königsinschriften des Dritten Jahrtausends v. Chr., in: FAOS 7, Stuttgart.

Hallo, William W. (1957): Early Mesopotamian Royal Titles: A Philological and Historical Analysis, in: American Oriental Series, 43, New Haven, Conn.

Heimpel, Wolfgang (1992): »Herrentum und Königtum«, in: Zeitschrift für Assyriologie und vorderasiatische Archäologie, 82, S. 4-21.

Jacobsen, Thorkild (1939): The Sumerian King List, in: Assyriological Studies 11, Chicago.

Jacobsen, Thorkild (1957): »Early political development in Mesopotamia«, in: Zeitschrift für Assyriologie und vorderasiatische Archäologie, 52, S. 91-140.

Jacobsen, Thorkild (1991): »The term ensi«, in: Aula Orientalis, 9, S. 113-121.

Kärki, Ilmari (1986): Die Inschriften der Dritten Dynastie von Ur, in: Studia Orientalia, 58 , Helsinki.

Kienast, Burkart (1973): »Der Weg zur Einheit Babyloniens unter staatsrechtlichen Aspekten«, in: Orientalia Nova Series, 42, S. 489-501.

Kienast, Burkart/Sommerfeld, W. (1994): Glossar zu den altakkadischen Königsinschriften, in: FAOS 8, Stuttgart. 
Krebernik, Manfred (1986): »Die Götterlisten aus Făra«, in: Zeitschrift für Assyriolgie und vorderasiatische Archäologie, 76, S. 161-204.

Kutscher, Raphael (1989): Royal Inscriptions: The Brockmon Tablets at the University of Haifa, Haifa.

Liverani, Mario (Hg.)(1993): Akkad. The First World Empire. Structure, Ideology, Traditions, Padova.

Mander, Pietro (1986): Il Pantheon di Abu-Șälabikh. Contributo allo studio del pantheon sumerico arcaico, Napoli.

Nissen, Hans J. (1983): Grundzüge einer Geschichte der Frühzeit des Vorderen Orients, Darmstadt.

Salonen, Armas (1965): Die Hausgeräte der alten Mesopotamier nach sumerischakkadischen Quellen, Helsinki.

Selz, Gebhard J. (1991): "Elam und Sumer - Skizze einer Nachbarschaft nach inschriftlichen Quellen der vorsargonischen Zeit «, in: Actes de la XXXVIème Rencontre Assyriologique Internationale, Gand, 10-14 juillet 1989.

Selz, Gebhard J. (1992): Enlil und Nippur nach präsargonischen Quellen, in: Nippur at the Centennial, Philadelphia, S. 189-225.

Selz, Gebhard J. (1995): Untersuchungen zur Götterwelt des altsumerischen Stadtstaates von Lagaš, in: Occasional Publications of the Samuel Noah Kramer Fund (= OPSNKF), 13, Philadelphia.

Selz, Gebhard J. (1998): „Über Mesopotamische Herrschaftskonzepte. Zu den Ursprüngen mesopotamischer Herrschaftsideologie im 3. Jahrtausend«, in: Manfried Dietrich/Oswald Loretz (Hg.), dubsar anta-men, Münster, S. 281-344.

Sjöberg, Ake W. (1972): »Die göttliche Abstammung der sumerisch-babylonischen Herrscher $\ll$, in: Orientalia Suecana, 21, S. 87-112.

Steible, Horst (1982): Die altsumerischen Bau- und Weihinschriften. Teil I: Inschriften aus >Lagaš, Teil II: Kommentar zu den Inschriften aus >Lagaš, Inschriften außerhalb von >Lagaš, in: FAOS 5, Wiesbaden.

Steible, Horst (1991): Die neusumerischen Bau- und Weihinschriften. Teil I: Inschriften der II. Dynastie von Lagas, Teil II: Kommentar zu den Gudea-Statuen, Inschriften der III. Dynastie von Ur, Inschriften der IV. und $>$ V.< Dynastie von Uruk, Varia, in: FAOS 9, Stuttgart.

Steible, Horst (1999): »Zu den Nahtstellen in den altmesopotamischen Codices $«$, in: Joachim Marzahn/Hans Neumann (Hg.), Assyriologica et Semitica. Festschrift für Joachim Oelsner (Alter Orient und Altes Testament, Bd. 252), Münster.

Steiner, Gerd (1986): »Der Grenzvertrag zwischen Lagaš und Umma«, in: Acta Sumerologica, 8, S. 219-300.

Steinkeller, Piotr (1982): »The question of Marbasi: A contribution to the historical geography of Iran in the third millenium B.C.«, in: Zeitschrift für Assyriologie und vorderasiatische Archäologie, 72, S. 237-265.

Steinkeller, Piotr (1993): »Early political development in Mesopotamia and the origins of the Sargonic empire«, in: Mario Liverani (Hg.), Akkad, Padova, S. 107129. 


\section{Tafel I}

Herrscher der altsumerischen, altakkadischen und neusumerischen Perioden

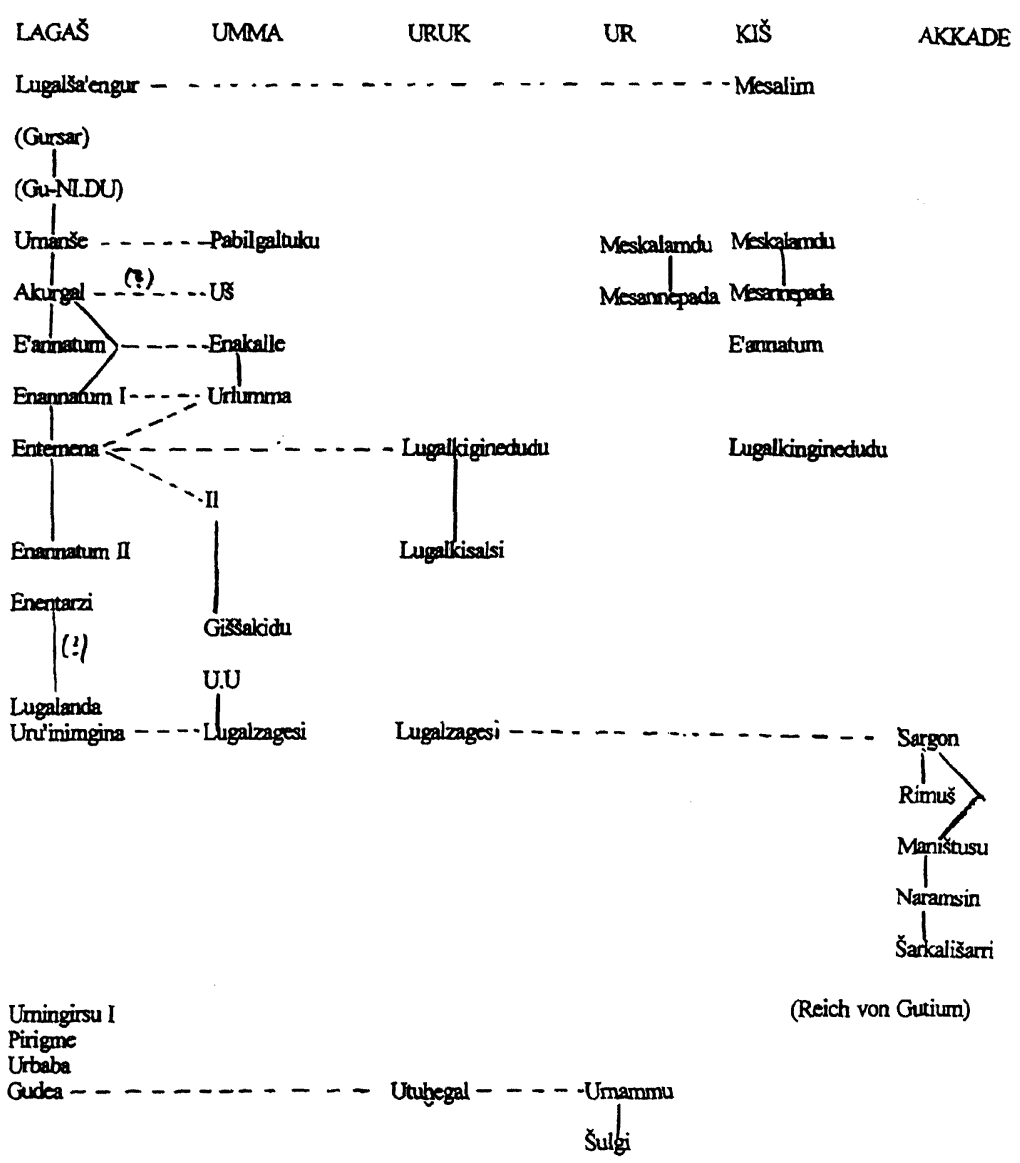




\section{Tafel II}

Sumerisches Pantheon des 3. Jahrtausends (nach J.S. Cooper, 1983, S. 61)
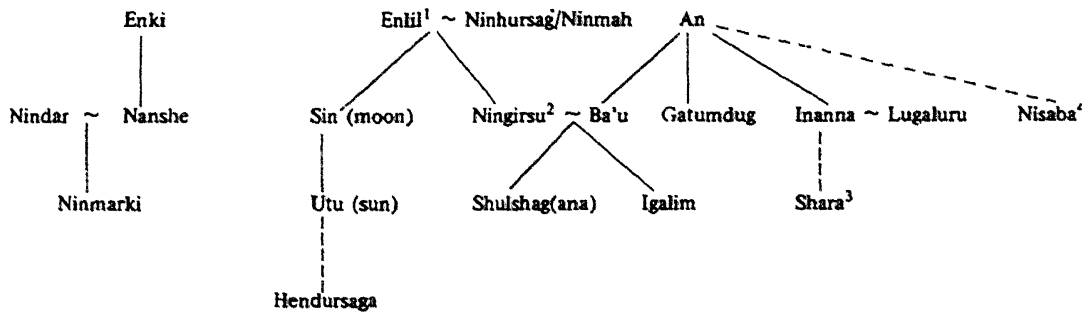

fliation

- - - possible filiation

'Ohief of Sumcrian puntheon.

2Chict of Lapasis punthion.

3Onicf of Unmen pantheos.

4 Chiet goddess of Umma. 
Karte Mesopotamiens in der Frühzeit (nach D.O. Edzard/G. Farber/E. Sollberger, 1977)

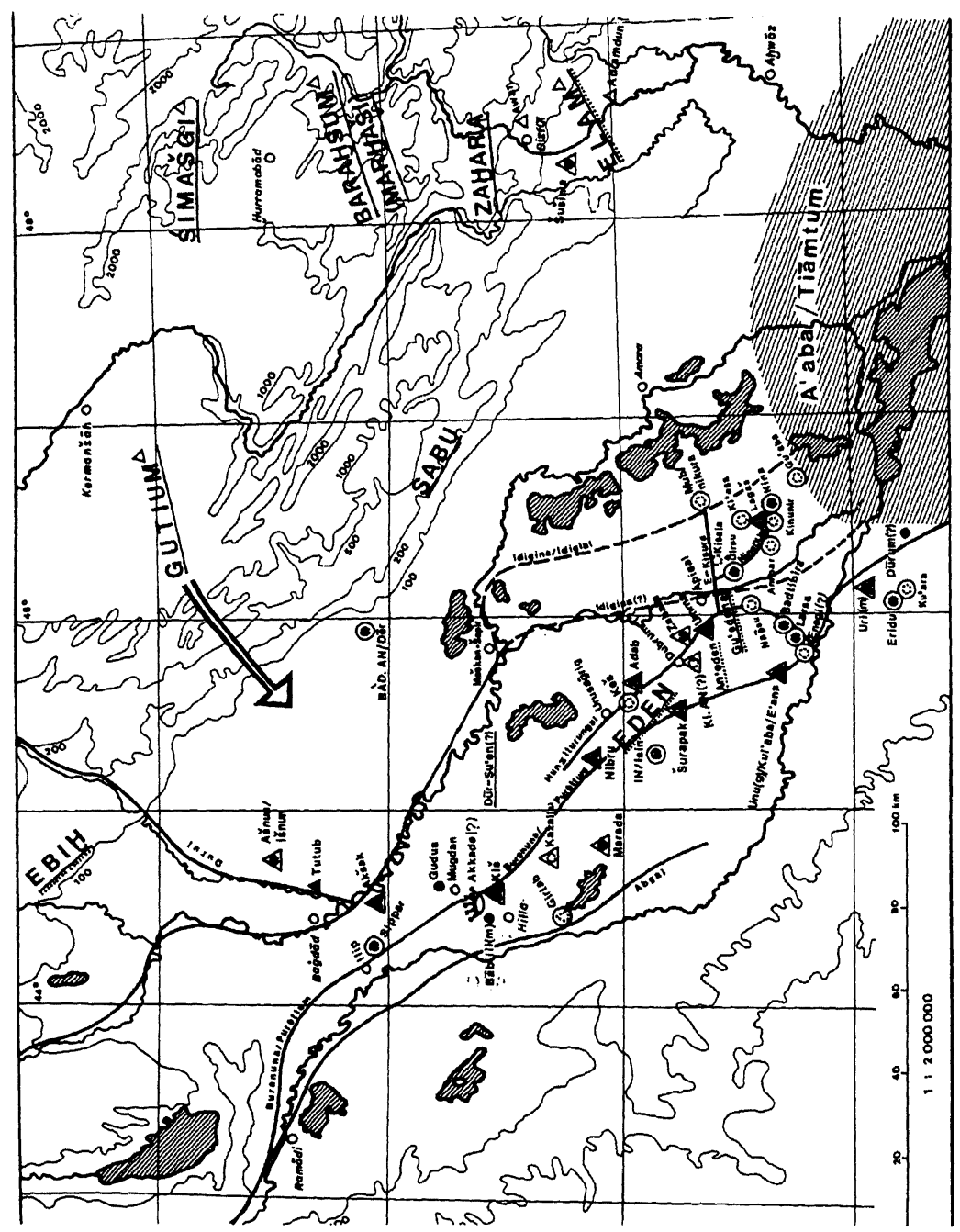

